\title{
Technical Note: Three-dimensional transient groundwater flow due to localized recharge with an arbitrary transient rate in unconfined aquifers
}

\author{
Chia-Hao Chang, Ching-Sheng Huang, and Hund-Der Yeh \\ Institute of Environmental Engineering, National Chiao Tung University, Hsinchu, Taiwan \\ Correspondence to: Hund-Der Yeh (hdyeh@mail.nctu.edu.tw)
}

Received: 18 September 2015 - Published in Hydrol. Earth Syst. Sci. Discuss.: 24 November 2015

Revised: 3 February 2016 - Accepted: 8 March 2016 - Published: 22 March 2016

\begin{abstract}
Most previous solutions for groundwater flow induced by localized recharge assumed either aquifer incompressibility or two-dimensional flow in the absence of the vertical flow. This paper develops a new three-dimensional flow model for hydraulic head variation due to localized recharge in a rectangular unconfined aquifer with four boundaries under the Robin condition. A governing equation describing spatiotemporal head distributions is employed. The first-order free-surface equation with a source term defining a constant recharge rate over a rectangular area is used to depict water table movement. The solution to the model for the head is developed with the methods of Laplace transform and double-integral transform. Based on Duhamel's theorem, the present solution is applicable to flow problems accounting for arbitrary time-dependent recharge rates. The solution to depth-average head can then be obtained by integrating the head solution to elevation and dividing the result by the aquifer thickness. The use of a rectangular aquifer domain has two merits. One is that the integration for estimating the depth-average head can be analytically achieved. The other is that existing solutions based on aquifers of infinite extent can be considered as special cases of the present solution before the time when the aquifer boundary had an effect on head predictions. With the help of the present solution, the assumption of neglecting the vertical flow effect on the temporal head distribution at an observation point outside a recharge region can be assessed by a dimensionless parameter related to the aquifer horizontal and vertical hydraulic conductivities, initial aquifer thickness, and the shortest distance between the observation point and the edge of the recharge region. The validity of assuming aquifer incompressibility is dominated by the ratio of the
\end{abstract}

aquifer specific yield to its storage coefficient. In addition, a sensitivity analysis is performed to investigate the head response to the change in each of the aquifer parameters.

\section{Introduction}

The water table rises due to localized recharge, such as rainfall, lakes, and agricultural irrigation, into the regional area of the aquifer. Excess recharge may cause soil liquefaction or wet basements of buildings. Groundwater flow behavior induced by recharge is therefore crucial in water resource management. The Boussinesq equation has been extensively used to describe horizontal flow without the vertical component in unconfined aquifers (e.g., Ireson and Butler, 2013; van der Spek et al., 2013; Yeh and Chang, 2013; Chor and Dias, 2015; Hsieh et al., 2015; Liang and Zhang, 2015; Liang et al., 2015). The equation can be linearized by assuming uniform saturated aquifer thickness for developing its analytical solution. Marino (1967) presented quantitative criteria for the validity of the linearized Boussinesq equation. The criteria are introduced in the next section.

The rate of localized recharge can be a constant for the long term but should be dependent of time for the short term (Rai et al., 2006). An exponentially decaying function of time is usually used for recharge intensity decreasing from a certain rate to an ultimate one. An arbitrary time-dependent recharge rate is commonly approximated as the combination of several linear segments of time to develop analytical solutions for water table rise subject to the recharge. 
Analytical models accounting for water table rise due to the recharge region of an infinite-length strip are reviewed. One-dimensional (1-D) flow perpendicular to the strip is considered while the flow along the strip is assumed ignorable. These models deal with aquifers of infinite or finite extent with various types of outer boundary conditions. Hantush (1963) considered an aquifer of infinite extent without a lateral boundary. Rao and Sarma (1980) considered an aquifer of finite extent with two constant-head (also called Dirichlet) boundaries. Later, they developed a solution (Rao and Sarma, 1984) for a finite-extent aquifer between no-flow and constant-head boundaries. Latinopoulos (1986) deliberated on a finite-extent aquifer between two boundaries, one of which is under the Robin condition and the other is under either the Dirichlet or no-flow condition. The recharge rate is treated as a periodical pulse consisting of constant rates for rainy seasons and zero for dry seasons. Bansal and Das (2010) studied an aquifer extending semi-infinitely from a Dirichlet boundary and overlying a sloping impervious base, and indicated that the change in groundwater mound induced by a strip-shaped recharge region increases with the base slope.

A variety of analytical models were presented to describe water table rise for 2-D flow induced by rectangle-shaped recharge into unconfined aquifers. The differences between these solutions are addressed below. Hantush (1967) considered an infinite-extent aquifer with localized recharge having a constant rate. Manglik et al. (1997) handled an arbitrary time-varying rate of recharge into a rectangular aquifer bounded by no-flow stratum. Manglik and Rai (1998) investigated flow behavior based on an irregularly time-varying rate of recharge into a rectangular aquifer with the lateral boundary under the Dirichlet condition. Bruggeman (1999) introduced an analytical solution for steady-state flow induced by localized recharge into a vertical strip aquifer between two Robin boundaries. Chang and Yeh (2007) considered one localized recharge and multiple extraction wells in an anisotropic aquifer overlying an impervious sloping bed. They indicated that the aquifer anisotropy and bottom slope notably influence water table distributions. Bansal and Teloglou (2013) explored the problem of a groundwater mound subject to multiple localized recharges and withdrawal wells in an unconfined aquifer overlying a semi-permeable base. They indicated that groundwater mound rises as the aquifer hydraulic conductivity decreases.

Some articles discussed water table rise near circle-shaped recharge regions and thus considered radial groundwater flow, which is symmetric to the center of the region. Rai et al. (1998) presented an analytical model describing water table growth subject to an exponentially decaying rate of recharge in a circle-shaped unconfined aquifer with an outer Dirichlet boundary. Illas et al. (2008) considered the same model but a leaky aquifer. They indicated that leakage across the aquifer bottom significantly influences spatiotemporal water table distributions despite a small amount of the leakage. On the other hand, some researchers considered radial flow having the vertical component near a circle-shaped recharge region of an infinite-extent unconfined aquifer. A first-order free-surface equation as the top boundary condition of the aquifer is applied to describe water table rise. Zlotnik and Ledder (1992) developed analytical models for describing the distributions of hydraulic head and flow velocity due to constant-rate recharge. They found that models neglecting aquifer compressibility overestimate the magnitudes of the head and flow velocity. Ostendorf et al. (2007) derived an analytical model for head variation due to an exponentially decaying rate of recharge. Predictions of their solution agreed well with the field data obtained in the Plymouth-Carver aquifer in southeastern Massachusetts given by Hansen and Lapham (1992).

Some studies developed a 3-D flow model based on the Laplace equation, which neglects the aquifer compressibility effect. Dagan (1967) derived an analytical solution for the velocity potential caused by regional recharge into an unconfined aquifer of infinite thickness. Zlotnik and Ledder (1993) also developed an analytical solution for the same model but considered finite thickness for the unconfined aquifer. Predictions of their solutions indicate that groundwater flow is horizontal in the area beyond $150 \%$ of the length or width of a rectangular recharge region.

It would be informative to summarize the abovementioned models in Table 1. The solutions to the models are classified according to flow dimensions into 1-D, 2-D, 3-D, and radial flows and further categorized according to aquifer domain, aquifer boundary conditions, recharge region, and recharge rate. The table shows that those solutions assume neither vertical flow nor aquifer incompressibility. In addition, the Dirichlet and no-flow conditions considered by some of those solutions are not applicable to a boundary having a semipermeable stratum, but the Robin condition is. The former two conditions are indeed special cases of the third one.

The objective of this paper is to develop a new mathematical model for depicting spatiotemporal hydraulic head distributions subject to localized recharge with an arbitrary time-varying recharge rate in a rectangular-shaped unconfined aquifer. The four boundaries are considered under the Robin condition, which can reduce to the Dirichlet or no-flow condition. A governing equation describing 3-D transient flow subject to the effect of aquifer compressibility is used. A first-order free-surface equation with a source term representing recharge rate is chosen to describe the top boundary condition. The transient head solution of the model is derived by the methods of Laplace transform, double-integral transform, and Duhamel's theorem. The sensitivity analysis based on the present solution is performed to study the head response to the change in each of the hydraulic parameters. On the basis of the solution's predictions, the effect of the Robin boundaries on time-dependent head distributions at observation points is investigated. A quantitative criterion under which the Robin condition reduces to the Dirichlet or no-flow 


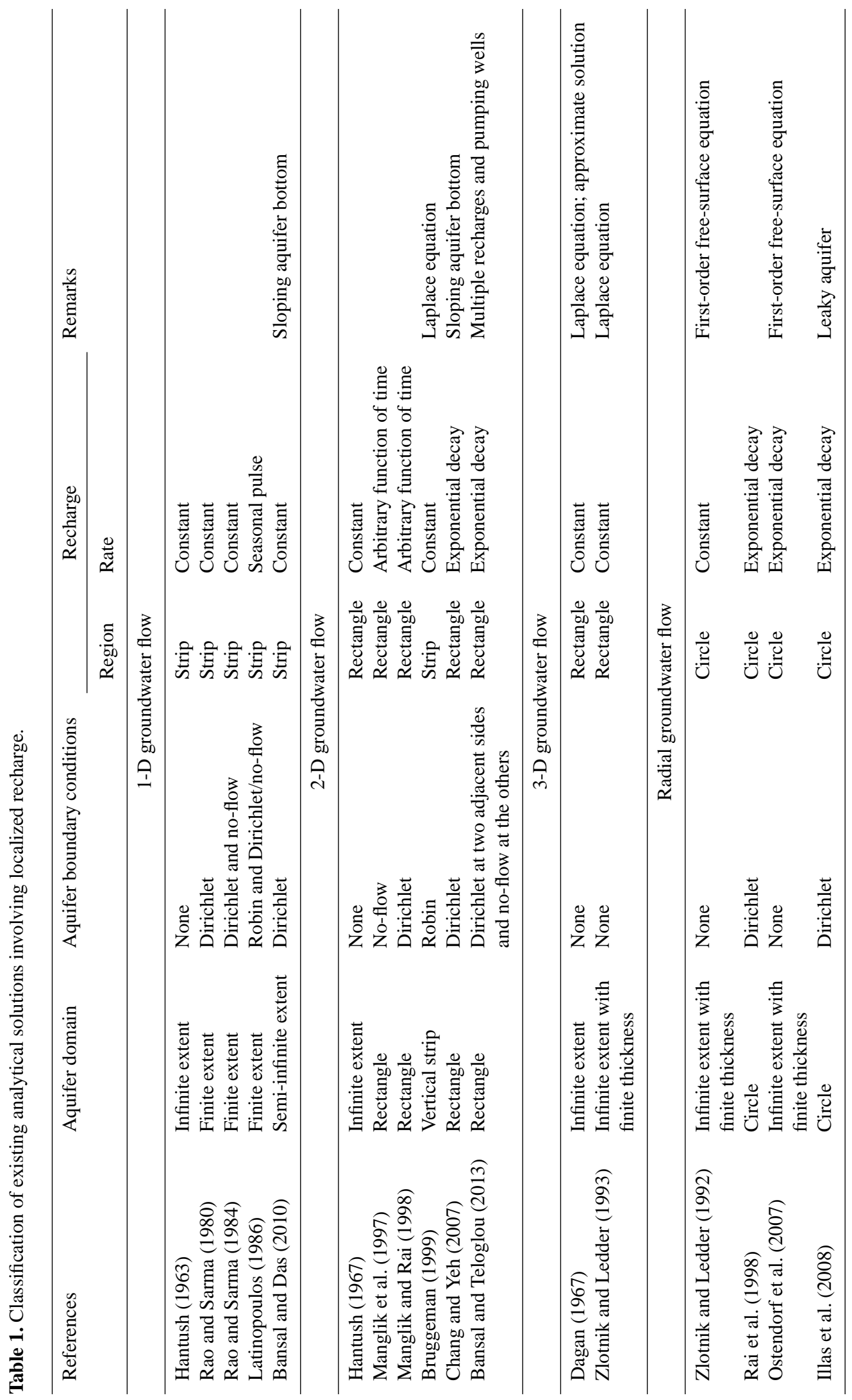


(a)

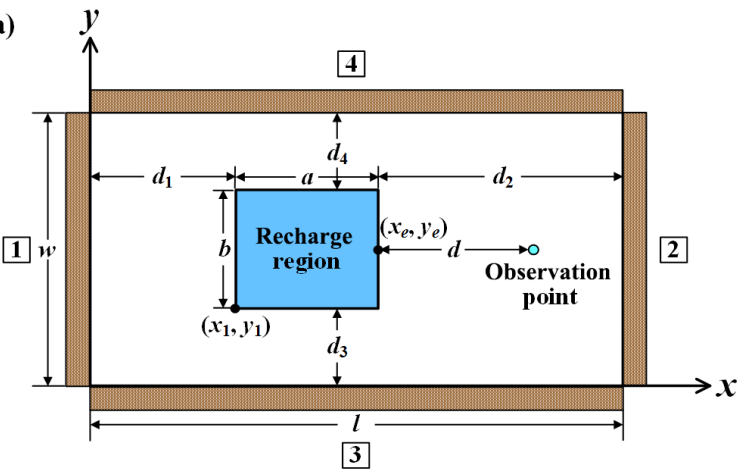

Robin boundary

Number of boundary (b)

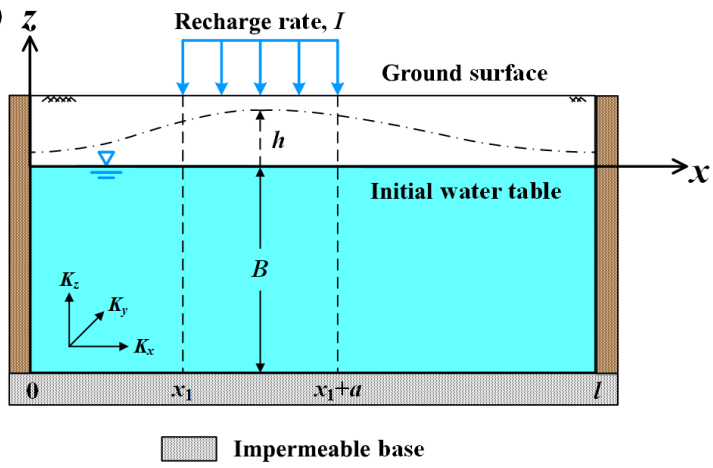

Figure 1. Schematic diagram of a rectangular-shaped unconfined aquifer with localized recharge (a) top view (b) cross section view.

condition is provided. In addition, quantitative criteria for the validity of two assumptions of aquifer incompressibility and no vertical flow are provided, and errors arising from the assumptions in the hydraulic head are also discussed. Temporal head distributions accounting for transient recharge rates are demonstrated as well.

\section{Methodology}

\subsection{Mathematical model}

A mathematical model is developed for describing spatiotemporal hydraulic head distributions induced by localized recharge in a rectangular unconfined aquifer as illustrated in Fig. 1a. The four boundaries of the aquifer are considered under the Robin condition. The aquifer has the widths of $l$ and $w$ in $x$ and $y$ directions, respectively. The recharge uniformly distributes over a rectangular region having widths $a$ and $b$ in $x$ and $y$ directions, respectively. The lower left corner of the region is designated at $\left(x_{1}, y_{1}\right)$. The shortest distances measured from the edge of the region to boundaries $1,2,3$, and 4 are denoted as $d_{1}, d_{2}, d_{3}$, and $d_{4}$, respectively. The shortest distance between the edge of the region and an observation point at $(x, y)$ is defined as $d=\min \left(\sqrt{\left(x-x_{\mathrm{e}}\right)^{2}+\left(y-y_{\mathrm{e}}\right)^{2}}\right)$, where $\left(x_{\mathrm{e}}, y_{\mathrm{e}}\right)$ is a coordinate on the edge. The initial aquifer's thickness is $B$ as shown in Fig. 1b.

The governing equation describing 3-D transient head distributions in a homogeneous and anisotropic aquifer is expressed as

$K_{x} \frac{\partial^{2} h}{\partial x^{2}}+K_{y} \frac{\partial^{2} h}{\partial y^{2}}+K_{z} \frac{\partial^{2} h}{\partial z^{2}}=S_{\mathrm{s}} \frac{\partial h}{\partial t}$,

where $t$ is time, $h(x, y, z, t)$ represents the hydraulic head, $K_{x}, K_{y}$, and $K_{z}$ are the hydraulic conductivities in $x, y$, and $z$ directions, respectively, and $S_{\mathrm{s}}$ is the specific storage. The initial static water table is chosen as the reference datum where the elevation head is set to zero. The initial condition is therefore written as

$h=0$ at $t=0$.

The Robin conditions specified at the four sides of the aquifer are defined as

$$
\begin{aligned}
& \frac{\partial h}{\partial x}-\frac{K_{1}}{K_{x} b_{1}} h=0 \text { at } x=0, \\
& \frac{\partial h}{\partial x}+\frac{K_{2}}{K_{x} b_{2}} h=0 \text { at } x=l, \\
& \frac{\partial h}{\partial y}-\frac{K_{3}}{K_{y} b_{3}} h=0 \text { at } y=0, \\
& \frac{\partial h}{\partial y}+\frac{K_{4}}{K_{y} b_{4}} h=0 \text { at } y=w,
\end{aligned}
$$

where subscripts $1,2,3$, and 4 represent the boundaries at $x=0, x=l, y=0$, and $y=w$, respectively, and $K$ and $b$ are the hydraulic conductivity and width of the medium at the aquifer boundary, respectively. Note that Eqs. (3)-(6) reduce to the Dirichlet condition when $b$ (i.e., $b_{1}, b_{2}, b_{3}$, or $b_{4}$ ) is set to zero and the no-flow condition when $K$ (i.e., $K_{1}, K_{2}$, $K_{3}$, or $K_{4}$ ) is set to zero. The aquifer lies on an impermeable base denoted as

$\partial h / \partial z=0$ at $z=-B$.

The first-order free-surface equation describing the response of water table to recharge over the rectangular region can be written as (Zlotnik and Ledder, 1993)

$K_{z} \frac{\partial h}{\partial z}+S_{\mathrm{y}} \frac{\partial h}{\partial t}=I u_{x} u_{y}$ at $z=0$,
$u_{x}=u\left(x-x_{1}\right)-u\left(x-x_{1}-a\right)$,
$u_{y}=u\left(y-y_{1}\right)-u\left(y-y_{1}-b\right)$,

where $S_{\mathrm{y}}$ is the specific yield, $I$ is a recharge rate, and $u()$ is the unit step function. Equation (8a) involves the assumption of $I \ll K_{z}$ and the simplification from non-uniform saturated aquifer thickness below $z=h$ to a uniform thickness 
below $z=0$ (Dagan, 1967). Marino (1967) indicated that the simplification and assumption are valid when the water table rise is smaller than $50 \%$ of the initial water table height (i.e., $|h| / B<0.5$ ) and the recharge rate is smaller than $20 \%$ of the hydraulic conductivity (i.e., $I / K_{z}<0.2$ ). On the other hand, the effect of unsaturated flow above the water table on the model's predictions can be ignored when $\sigma B \geq 10^{3}$, where $\sigma$ is a parameter to define the relative hydraulic conductivity as $k_{0}=\exp (-\sigma z)$ in the Richards' equation (Tartakovsky and Neuman, 2007). Tartakovsky and Neuman (2007) achieved agreement on aquifer drawdown, which was evaluated by their analytical solution based on Eq. (1) for saturated flow and Richards' equation for unsaturated flow, and by the Neuman (1974) solution based on Eqs. (1) and (8a) with $I=0$ when $\sigma B=10^{3}$ (i.e., the case of $\kappa_{D}=10^{3}$ in Fig. 2 in Tartakovsky and Neuman, 2007).

Dimensionless variables and parameters are defined as follows

$\bar{h}=\frac{h}{B}, \bar{x}=\frac{x}{d}, \bar{y}=\frac{y}{d}, \bar{z}=\frac{z}{B}, \bar{l}=\frac{l}{d}, \bar{w}=\frac{w}{d}$,

$\bar{x}_{1}=\frac{x_{1}}{d}, \bar{y}_{1}=\frac{y_{1}}{d}, \bar{a}=\frac{a}{d}, \bar{b}=\frac{b}{d}, \kappa_{z}=\frac{K_{z} d^{2}}{K_{x} B^{2}}$,

$\bar{t}=\frac{K_{x} t}{S_{\mathrm{s}} d^{2}}, \kappa_{y}=\frac{K_{y}}{K_{x}}, \kappa_{1}=\frac{K_{1} d}{K_{x} b_{1}}, \kappa_{2}=\frac{K_{2} d}{K_{x} b_{2}}$,

$\kappa_{3}=\frac{K_{3} d}{K_{y} b_{3}}, \kappa_{4}=\frac{K_{4} d}{K_{y} b_{4}}, \xi=\frac{I}{K_{z}}, \varepsilon=\frac{S_{\mathrm{y}}}{S_{\mathrm{s}} B}, \bar{d}_{1}$

$=\frac{d_{1}}{d}, \bar{d}_{2}=\frac{d_{2}}{d}, \bar{d}_{3}=\frac{d_{3}}{d}, \bar{d}_{4}=\frac{d_{4}}{d}$,

where the overbar denotes a dimensionless symbol. Notice that the variables in the horizontal and vertical directions are divided by $d$ and $B$, respectively. According to Eq. (9), the mathematical model, Eqs. (1)-(8c), can then be expressed as

$\frac{\partial^{2} \bar{h}}{\partial \bar{x}^{2}}+\kappa_{y} \frac{\partial^{2} \bar{h}}{\partial \bar{y}^{2}}+\kappa_{z} \frac{\partial^{2} \bar{h}}{\partial \bar{z}^{2}}=\frac{\partial \bar{h}}{\partial \bar{t}}$,

$\bar{h}=0$ at $\bar{t}=0$,

$\frac{\partial \bar{h}}{\partial \bar{x}}-\kappa_{1} \bar{h}=0$ at $\bar{x}=0$,

$\frac{\partial \bar{h}}{\partial \bar{x}}+\kappa_{2} \bar{h}=0$ at $\bar{x}=\bar{l}$,

$\frac{\partial \bar{h}}{\partial \bar{y}}-\kappa_{3} \bar{h}=0$ at $\bar{y}=0$,

$\frac{\partial \bar{h}}{\partial \bar{y}}+\kappa_{4} \bar{h}=0$ at $\bar{y}=\bar{w}$

$\partial \bar{h} / \partial \bar{z}=0$ at $\bar{z}=-1$,

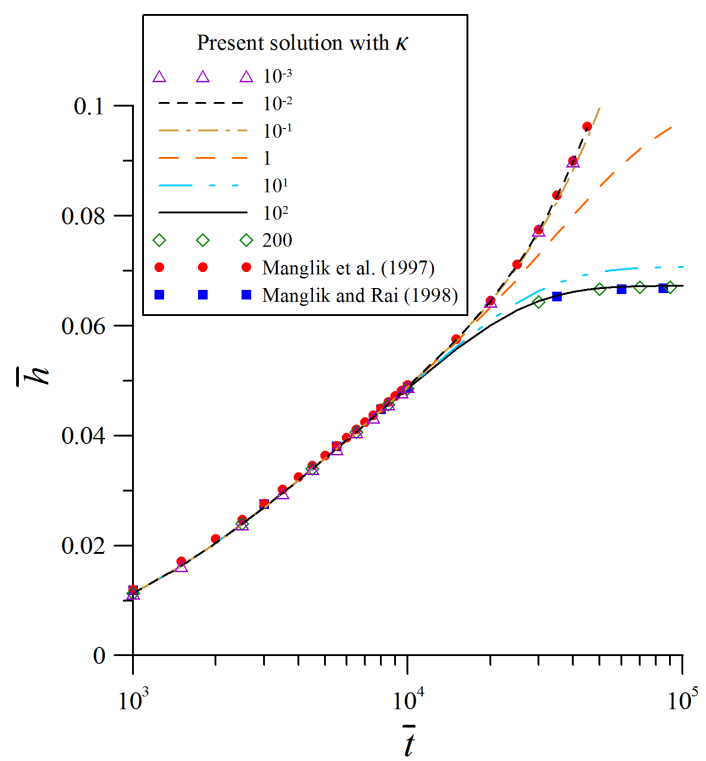

Figure 2. Temporal distributions of the dimensionless head predicted by the Manglik et al. (1997) solution for a no-flow boundary, the Manglik and Rai (1998) solution for a Dirichlet boundary, and the present solution with $\kappa_{z}=1$ for a Robin boundary.

$$
\begin{aligned}
& \frac{\partial \bar{h}}{\partial \bar{z}}+\frac{\varepsilon}{\kappa_{z}} \frac{\partial \bar{h}}{\partial \bar{t}}=\xi \bar{u}_{x} \bar{u}_{y} \text { at } \bar{z}=0, \\
& \bar{u}_{x}=u\left(\bar{x}-\bar{x}_{1}\right)-u\left(\bar{x}-\bar{x}_{1}-\bar{a}\right), \\
& \bar{u}_{y}=u\left(\bar{y}-\bar{y}_{1}\right)-u\left(\bar{y}-\bar{y}_{1}-\bar{b}\right) .
\end{aligned}
$$

\subsection{Analytical solution}

The mathematical model, Eqs. (10)-(17c), can be solved by the methods of Laplace transform and double-integral transform. The former transform converts $\bar{h}(\bar{x}, \bar{y}, \bar{z}, \bar{t})$ into $\widetilde{h}(\bar{x}$, $\bar{y}, \bar{z}, p), \partial \bar{h} / \partial \bar{t}$ into $p \widetilde{h}-\left.\bar{h}\right|_{\bar{t}=0}$, and $\xi \bar{u}_{x} \bar{u}_{y}$ into $\xi \bar{u}_{x} \bar{u}_{y} / p$, where $p$ is the Laplace parameter and $\left.\bar{h}\right|_{\bar{t}=0}$ equals zero in Eq. (11). After taking the transform, the model becomes a boundary value problem expressed as

$\frac{\partial^{2} \widetilde{h}}{\partial \bar{x}^{2}}+\kappa_{y} \frac{\partial^{2} \widetilde{h}}{\partial \bar{y}^{2}}+\kappa_{z} \frac{\partial^{2} \widetilde{h}}{\partial \bar{z}^{2}}=p \widetilde{h}$,

with boundary conditions $\partial \widetilde{h} / \partial \bar{x}-\kappa_{1} \widetilde{h}=0$ at $\bar{x}=0$, $\partial \widetilde{h} / \partial \bar{x}+\kappa_{2} \widetilde{h}=0 \quad$ at $\bar{x}=\bar{l}, \quad \partial \widetilde{h} / \partial y-\kappa_{3} \widetilde{h}=0 \quad$ at $\bar{y}=0$, $\partial \widetilde{h} / \partial \bar{y}+\kappa_{4} \widetilde{h}=0$ at $\bar{y}=\bar{w}, \partial \widetilde{h} / \partial \bar{z}=0$ at $\bar{z}=-1$, and $\partial \widetilde{h} / \partial \bar{z}+\varepsilon p \widetilde{h} / \kappa_{z}=\xi \bar{u}_{x} \bar{u}_{y} / p$ at $\bar{z}=0$. We then apply the properties of the double-integral transform to the problem. One can refer to the definition in Latinopoulos (1985, Table I, aquifer type 1$)$. The transform turns $\widetilde{h}(\bar{x}, \bar{y}, \bar{z}, p)$ into $\hat{h}\left(\alpha_{m}\right.$, $\left.\beta_{n}, \bar{z}, p\right), \partial^{2} \widetilde{h} / \partial \bar{x}^{2}+\kappa_{y}\left(\partial^{2} \widetilde{h} / \partial \bar{y}^{2}\right)$ into $-\left(\alpha_{m}^{2}+\kappa_{y} \beta_{n}^{2}\right) \hat{h}$, where $(m, n) \in 1,2,3, \ldots \infty$, and eigenvalues $\alpha_{m}$ and $\beta_{n}$ are the positive roots of the following equations: 
$\tan \left(\bar{l} \alpha_{m}\right)=\frac{\alpha_{m}\left(\kappa_{1}+\kappa_{2}\right)}{\alpha_{m}^{2}-\kappa_{1} \kappa_{2}}$

and

$\tan \left(\bar{w} \beta_{n}\right)=\frac{\beta_{n}\left(\kappa_{3}+\kappa_{4}\right)}{\beta_{n}^{2}-\kappa_{3} \kappa_{4}}$.

In addition, $\bar{u}_{x} \bar{u}_{y}$ defined in Eqs. (17b) and (17c) is transformed into $U_{m} U_{n}$ given by

$U_{m}=\frac{\sqrt{2} V_{m}}{\sqrt{\kappa_{1}+\left(\alpha_{m}^{2}+\kappa_{1}^{2}\right)\left[\bar{l}+\kappa_{2} /\left(\alpha_{m}^{2}+\kappa_{2}^{2}\right)\right]}}$,
$U_{n}=\frac{\sqrt{2} V_{n}}{\sqrt{\kappa_{3}+\left(\beta_{n}^{2}+\kappa_{3}^{2}\right)\left[\bar{w}+\kappa_{4} /\left(\beta_{n}^{2}+\kappa_{4}^{2}\right)\right]}}$,

with

$$
\begin{aligned}
V_{m} & =\left\{\kappa_{1}\left[\cos \left(\alpha_{m} \bar{x}_{1}\right)-\cos \left(\alpha_{m} \chi\right)\right]\right. \\
& \left.-\alpha_{m}\left[\sin \left(\alpha_{m} \bar{x}_{1}\right)-\sin \left(\alpha_{m} \chi\right)\right]\right\} / \alpha_{m},
\end{aligned}
$$

$$
\begin{aligned}
V_{n} & =\left\{\kappa_{3}\left[\cos \left(\beta_{n} \bar{y}_{1}\right)-\cos \left(\beta_{n} \psi\right)\right]\right. \\
& \left.-\beta_{n}\left[\sin \left(\beta_{n} \bar{y}_{1}\right)-\sin \left(\beta_{n} \psi\right)\right]\right\} / \beta_{n},
\end{aligned}
$$

where $\chi=\bar{x}_{1}+\bar{a}$ and $\psi=\bar{y}_{1}+\bar{b}$.

Equation (18) then reduces to an ordinary differential equation as

$\kappa_{z} \frac{\partial^{2} \hat{h}}{\partial \bar{z}^{2}}-\left(p+\alpha_{m}^{2}+\kappa_{y} \beta_{n}^{2}\right) \hat{h}=0$.

Two boundary conditions are expressed, respectively, as

$\partial \hat{h} / \partial \bar{z}=0$ at $\bar{z}=-1$

and

$\frac{\partial \hat{h}}{\partial \bar{z}}+\frac{\varepsilon p}{\kappa_{z}} \hat{h}=\frac{\xi}{p} U_{m} U_{n}$ at $\bar{z}=0$.

Solving Eq. (25) with Eqs. (26) and (27) results in

$\hat{h}\left(\alpha_{m}, \beta_{n}, \bar{z}, p\right)=\frac{\xi U_{m} U_{n} \cosh [(1+\bar{z}) \lambda]}{p\left(p \varepsilon \kappa_{z} \cosh \lambda+\kappa_{z} \lambda \sinh \lambda\right)}$,

where

$\lambda=\sqrt{\left(p+\alpha_{m}^{2}+\kappa_{y} \beta_{n}^{2}\right) / \kappa_{z}}$.

Inverting Eq. (28) to the space and time domains gives rise to the following analytical solution:

$$
\begin{gathered}
\bar{h}(\bar{x}, \bar{y}, \bar{z}, \bar{t})=\xi \sum_{m=1}^{\infty} \sum_{n=1}^{\infty}\left(\phi_{m, n}+\phi_{0, m, n}+\sum_{j=1}^{\infty} \phi_{j, m, n}\right) \\
F_{m} F_{n} U_{m} U_{n}
\end{gathered}
$$

with

$$
\begin{aligned}
& \phi_{m, n}=\frac{\cosh \left[(1+\bar{z}) \lambda_{m, n}\right]}{\kappa_{z} \lambda_{m, n} \sinh \lambda_{m, n}}, \\
& \phi_{0, m, n}=-2 \lambda_{0, m, n} \cosh \left[(1+\bar{z}) \lambda_{0, m, n}\right] \exp \left(-\gamma_{0, m, n} \bar{t}\right) / \eta_{0, m, n}, \\
& \phi_{j, m, n}=-2 \lambda_{j, m, n} \cos \left[(1+\bar{z}) \lambda_{j, m, n}\right] \exp \left(-\gamma_{j, m, n} \bar{t}\right) / \eta_{j, m, n}, \\
& \eta_{0, m, n}=\gamma_{0, m, n}\left[\left(1+2 \varepsilon \kappa_{z}\right) \lambda_{0, m, n} \cosh \lambda_{0, m, n}+\left(1-\varepsilon \gamma_{0, m, n}\right) \sinh \lambda_{0, m, n}\right], \\
& \eta_{j, m, n}=\gamma_{j, m, n}\left[\left(1+2 \varepsilon \kappa_{z}\right) \lambda_{j, m, n} \cos \lambda_{j, m, n}+\left(1-\varepsilon \gamma_{j, m, n}\right) \sin \lambda_{j, m, n}\right], \\
& \lambda_{m, n}=\sqrt{f_{m, n} / \kappa_{z}} ; \gamma_{0, m, n}=f_{m, n}-\kappa_{z} \lambda_{0, m, n}^{2} ; \gamma_{j, m, n}=f_{m, n}+\kappa_{z} \lambda_{j, m, n}^{2}, \\
& f_{m, n}=\alpha_{m}^{2}+\kappa_{y} \beta_{n}^{2}, \\
& F_{m}=\frac{\sqrt{2}\left[\alpha_{m} \cos \left(\alpha_{m} \bar{x}\right)+\kappa_{1} \sin \left(\alpha_{m} \bar{x}\right)\right]}{\sqrt{\kappa_{1}+\left(\alpha_{m}^{2}+\kappa_{1}^{2}\right)\left[\bar{l}+\kappa_{2} /\left(\alpha_{m}^{2}+\kappa_{2}^{2}\right)\right]},} \\
& F_{n}=\frac{\sqrt{2}\left[\beta_{n} \cos \left(\beta_{n} \bar{y}\right)+\kappa_{3} \sin \left(\beta_{n} \bar{y}\right)\right]}{\sqrt{\kappa_{3}+\left(\beta_{n}^{2}+\kappa_{3}^{2}\right)\left[\bar{w}+\kappa_{4} /\left(\beta_{n}^{2}+\kappa_{4}^{2}\right)\right]}},
\end{aligned}
$$

where $j \in 1,2,3, \ldots \infty$ and eigenvalues $\lambda_{0, m, n}$ and $\lambda_{j, m, n}$ are determined, respectively, by the following equations:

$\tan \lambda_{j, m, n}=-\varepsilon\left(f_{m, n}+\kappa_{z} \lambda_{j, m, n}^{2}\right) / \lambda_{j, m, n}$

and

$$
\frac{-\varepsilon \kappa_{z} \lambda_{0, m, n}^{2}+\lambda_{0, m, n}+\varepsilon f_{m, n}}{\varepsilon \kappa_{z} \lambda_{0, m, n}^{2}+\lambda_{0, m, n}-\varepsilon f_{m, n}}=\exp \left(2 \lambda_{0, m, n}\right) .
$$

Notice that Eqs. (19), (20), and (31) have infinite positive roots owing to the trigonometric function $\tan ()$ while Eq. (32) has only one positive root. The method to find $\alpha_{m}$, $\beta_{n}, \lambda_{j, m, n}$, and $\lambda_{0, m, n}$ is introduced in Sect. 2.3. One can refer to Appendix A for the derivation of Eq. (30a). The first term on the right-hand side (RHS) of Eq. (30a) is a double series expanded by $\alpha_{m}$ and $\beta_{n}$. The series converges within a few terms because the power of $\alpha_{m}$ (or $\beta_{n}$ ) in the denominator of $\phi_{m, n}$ in Eq. (30b) is two more than that in the nominator. The second term on the RHS of Eq. (30a) is a double series expanded by $\alpha_{m}$ and $\beta_{n}$, and the third term is a triple series expanded by $\alpha_{m}, \beta_{n}$, and $\lambda_{j, m, n}$. They converge very fast due to exponential functions in Eqs. (30c) and (30d). Consider $(m, n) \in(1,2, \ldots, N=30)$ and $j \in\left(1,2, \ldots, N_{j}=15\right)$ for the default values of dimensionless parameters and variables in Table A1 for calculation. The number of terms in one or the other double series is $30 \times 30=900$ and in the triple series is $30 \times 30 \times 15=13500$. The total number is therefore $900 \times 2+13500=15300$. We apply Mathematica FindRoot routine to obtain the values of $\alpha_{m}, \beta_{n}$, and $\lambda_{j, m, n}$ and Sum routine to compute the double and triple series. It 
takes about $8 \mathrm{~s}$ to finish calculation for $\bar{t}=10^{5}$ with a personal computer with Intel Core i5-4590 $3.30 \mathrm{GHz}$ processor and 8 GB RAM. In addition, the series is considered to converge when the absolute value of the last term in the double series of $\phi_{m, n}$ is smaller than $10^{-20}$ (i.e., $10^{-50}<10^{-20}$ in this case). That value in the other double or triple series may be even smaller than $10^{-50}$ due to exponential decay.

The use of finite aquifer domain has two merits. One is that the solution to depth-average head, defined as $\int_{-1}^{0} \bar{h}(\bar{x}$, $\bar{y}, \bar{z}, \bar{t}) \mathrm{d} \bar{z}$, can be analytically integrated. The integration variable $\bar{z}$ appears only in the functions of $\cosh [(1+$ $\left.\bar{z}) \lambda_{m, n}\right]$ in Eq. (30b), $\cosh \left[(1+\bar{z}) \lambda_{0, m, n}\right]$ in Eq. (30c) and $\cos \left[(1+\bar{z}) \lambda_{j, m, n}\right]$ in Eq. (30d). The solution to depthaverage head therefore equals Eq. (30a), where these three functions are replaced by $\sinh \lambda_{m, n} / \lambda_{m, n}, \sinh \lambda_{0, m, n} / \lambda_{0, m, n}$, and $\sin \lambda_{j, m, n} / \lambda_{j, m, n}$, respectively. The other merit is that the present solution is applicable to head predictions in aquifers of infinite extent before the dimensionless time to have lateral aquifer boundary effect on the predictions. Wang and Yeh (2008) reported a time criterion defined as $\bar{t}_{\text {cr }}=0.03(1+\varepsilon) \bar{R}^{2}$, where $\bar{R}=R / d$ denotes a shortest dimensionless distance from the lateral boundary to the edge of the recharge region. This criterion is, in effect, a boundaryeffect time when the hydraulic head is affected by the aquifer boundary. Existing solutions based on aquifers of infinite extent can therefore be considered as special cases of the present solution before the boundary-effect time.

\subsection{Calculation of eigenvalues}

The eigenvalues $\alpha_{m}, \beta_{n}, \lambda_{j, m, n}$, and $\lambda_{0, m, n}$ can be determined by Newton's method with initial guess values (IGVs) set to be the vertical asymptotes of the functions on the left-hand side (LHS) of Eqs. (19), (20), (31), and (32), respectively. Hence, IGVs for $\alpha_{m}$ are $\alpha^{\prime}+\delta$ if $\alpha^{\prime}<\left(\kappa_{1} \kappa_{2}\right)^{1 / 2}$ and $\alpha^{\prime}-\delta$ if $\alpha^{\prime}>\left(\kappa_{1} \kappa_{2}\right)^{1 / 2}$, where $\alpha^{\prime}=(2 m-1) \pi /(2 \bar{l})$ and $\delta$ is a small value of $10^{-8}$ to avoid being right at the vertical asymptotes. Similarly, IGVs for $\beta_{n}$ are $\beta^{\prime}+\delta$ if $\beta^{\prime}<\left(\kappa_{3} \kappa_{4}\right)^{1 / 2}$ and $\beta^{\prime}-\delta$ if $\beta^{\prime}>\left(\kappa_{3} \kappa_{4}\right)^{1 / 2}$, where $\beta^{\prime}=(2 n-1) \pi /(2 \bar{w})$. In addition, IGVs for $\lambda_{j, m, n}$ are $(2 j-1) \pi / 2+\delta$, and the IGV for $\lambda_{0, m, n}$ is $\delta+\left[\left(1+4 \kappa_{z} f_{m, n} \varepsilon^{2}\right)^{1 / 2}-1\right] /\left(2 \varepsilon \kappa_{z}\right)$ obtained by setting the denominator of the LHS function of Eq. (32) to zero and solving the resultant equation.

\subsection{Solution for time-varying recharge rate}

The present solution, Eq. (30a), is applicable to arbitrary time-dependent recharge rates on the basis of Duhamel's theorem expressed as (e.g., Bear, 1979, p. 158)
$\bar{h}_{I t}=\bar{h}_{I 0}+\int_{0}^{\bar{t}} \frac{\partial \xi_{t}(\tau)}{\partial \tau} \bar{h}(\bar{t}-\tau) / \xi \mathrm{d} \tau$,

where $\bar{h}_{I t}$ signifies a dimensionless head solution for a timedependent recharge rate $\xi_{t}(\tau)$ with $\bar{t}$ replaced by $\tau, \bar{h}_{I 0}$ is Eq. (30a) in which $\xi$ is replaced by $\xi_{t}(0)$, and $\bar{h}(\bar{t}-\tau)$ is also Eq. (30a) with $\bar{t}$ replaced by $\bar{t}-\tau$. If Eq. (33) is not integrable, it can be discretized as (Singh, 2005)

$\bar{h}_{N}=\sum_{i=1}^{N} \frac{\Delta \xi_{i}}{\Delta \bar{t}} \eta(N-i+1)$,

with

$\Delta \xi_{i}=\xi_{i}-\xi_{i-1}$,

$\eta(M)=\int_{0}^{\bar{t}} \bar{h}(M \Delta \bar{t}-\tau) d \tau$,

where $\bar{h}_{N}$ represents a numerical result of dimensionless head $\bar{h}$ at $\bar{t}=\Delta \bar{t} \times N, \Delta \bar{t}$ is a dimensionless time step, $\xi_{i}$ and $\xi_{i-1}$ are dimensionless recharge rates at $\bar{t}=\Delta \bar{t} \times i$ and $\bar{t}=\Delta \bar{t} \times(i-1)$, respectively, and $\eta(M)$, called ramp kernel, depends on Eq. (30a) in which $\bar{t}$ is replaced by $M \Delta \bar{t}-\tau$. The integration result of Eq. (34c) can be denoted as Eq. (30a) where $\phi_{m, n}$ is replaced by $\phi_{m, n} \bar{t}$ and two exponential terms in Eqs. (30c) and (30d) are replaced, respectively, by $\exp \left(-M \gamma_{0, m, n} \Delta \bar{t}\right)\left[-1+\exp \left(\gamma_{0, m, n} \Delta \bar{t}\right)\right] / \gamma_{0, m, n}$ and $\exp \left(-M \gamma_{j, m, n} \Delta \bar{t}\right)\left[-1+\exp \left(\gamma_{j, m, n} \Delta \bar{t}\right)\right] / \gamma_{j, m, n}$.

\subsection{Sensitivity analysis}

The sensitivity analysis is administered to assess the change in the hydraulic head in response to the change in each of the hydraulic parameters. The normalized sensitivity coefficient of the hydraulic head to a specific parameter can be expressed as

$S_{c, t}=\frac{\partial h / B}{\partial P_{c} / P_{c}}=\frac{\partial \bar{h}}{\partial P_{c} / P_{c}}$,

where $P_{c}$ is the $c$ th parameter in the present solution, $S_{c, t}$ is the coefficient at a time to the $c$ th parameter, and $\bar{h}$ is the present solution, Eq. (30a). The derivative in Eq. (35) can be approximated as

$S_{c, t}=\frac{\bar{h}\left(P_{c}+\Delta P_{c}\right)-\bar{h}\left(P_{c}\right)}{\Delta P_{c} / P_{c}}$,

where $\Delta P_{c}$ is an increment chosen as $10^{-3} P_{c}$ (Yeh et al., 2008). 


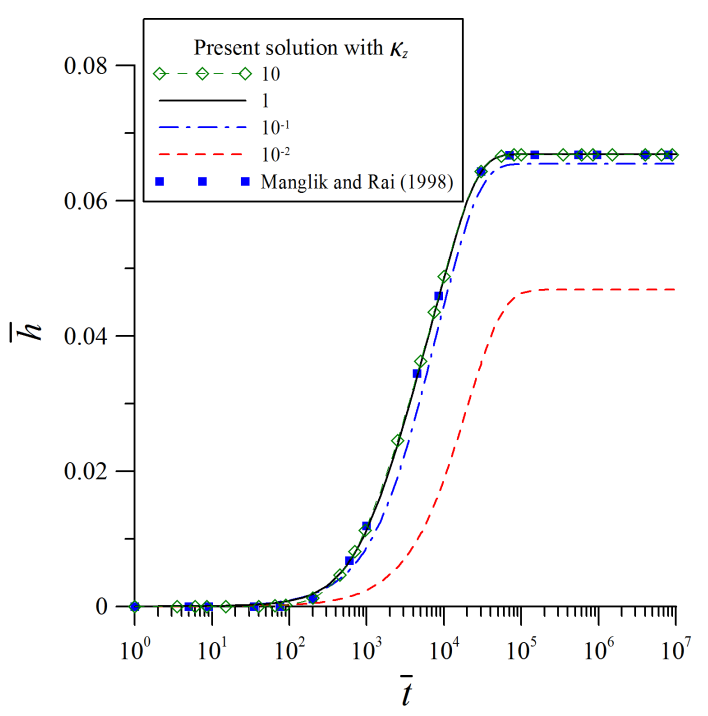

Figure 3. Temporal distributions of the dimensionless head predicted by the Manglik and Rai (1998) solution based on 2-D flow and the present solution for 3-D flow with various $\kappa_{z}$.

\section{Results and discussion}

Previous articles have discussed groundwater mounds in response to localized recharge into aquifers with various hydraulic parameters (e.g., Dagan, 1967; Rao and Sarma, 1980; Latinopoulos, 1986; Manglik et al., 1997; Manglik and Rai, 1998; Rai et al., 1998; Chang and Yeh, 2007; Illas et al., 2008; Bansal and Das, 2010; Bansal and Teloglou, 2013). Flow velocity fields below groundwater mounds have also been analyzed (Zlotnik and Ledder, 1992, 1993). This section therefore focuses on the transient behavior of hydraulic head at an observation point with the aid of the present solution. The default values of the parameters and variables for calculation are noted in Table A1. In Sect. 3.1, transient head distributions subject to Dirichlet, no-flow and Robin boundary conditions are compared. In Sect. 3.2, the effect of vertical flow on the head distribution is investigated. In Sect. 3.3, errors arising from assuming aquifer incompressibility (i.e., $S_{\mathrm{s}}=0$ ) to develop analytical solutions are discussed. In Sect. 3.4, the response of the hydraulic head to transient recharge rates based on Eq. (33) is demonstrated. In Sect. 3.5, the sensitivity analysis defined by Eq. (36) is performed.

\subsection{Effect of lateral boundary}

The Robin condition can become the Dirichlet or no-flow condition, depending on the magnitudes of $\kappa_{1} \bar{d}_{1}$ for Eq. (12), $\kappa_{2} \bar{d}_{2}$ for Eq. (13), $\kappa_{3} \bar{d}_{3}$ for Eq. (14), and $\kappa_{4} \bar{d}_{4}$ for Eq. (15). We consider a symmetrical aquifer system with $\bar{l}=\bar{w}=22$, $\bar{d}_{1}=\bar{d}_{2}=\bar{d}_{3}=\bar{d}_{4}=10$, and $\kappa_{1}=\kappa_{2}=\kappa_{3}=\kappa_{4}$. The magnitudes of $\kappa_{1} \bar{d}_{1}, \kappa_{2} \bar{d}_{2}, \kappa_{3} \bar{d}_{3}$, and $\kappa_{4} \bar{d}_{4}$ are the same and defined as $\kappa$. The curves of $\bar{h}$ versus $\bar{t}$ plotted by the present solution, Eq. (30a), for $\kappa=10^{-3}, 10^{-2}, 10^{-1}, 1,10,100$, and 200 are shown in Fig. 2. The curves $\bar{h}$ versus $\bar{t}$ are plotted from the Manglik et al. (1997) solution with the noflow condition (i.e., $\kappa=0$ ), the Manglik and Rai (1998) solution with the Dirichlet condition (i.e., $\kappa \rightarrow \infty$ ), and the present solution with the Robin condition. Before $\bar{t}=10^{4}$, these curves give the same magnitude of $\bar{h}$ at a fixed dimensionless time $\bar{t}$, since the lateral aquifer boundary has been beyond the place where groundwater is affected by localized recharge. After $\bar{t}=10^{4}$, the curves for the cases of $\kappa=10^{-2}$, $10^{-1}, 1,10$, and 100 deviate from each other gradually as time increases. A larger magnitude of $\kappa$ between $\kappa=10^{-2}$ and $\kappa=100$ causes a smaller $\bar{h}$ at a fixed $\bar{t}$. On the other hand, the present solution for the cases of $\kappa=10^{-3}$ and $10^{-2}$ agrees well with the Manglik et al. (1997) solution based on $\kappa=0$ and that for the cases of $\kappa=100$ and 200 predicts the same result as the Manglik and Rai (1998) solution based on $\kappa \rightarrow \infty$. We may reasonably conclude that the Robin condition reduces to the no-flow condition when $\kappa \leq 10^{-2}$ and the Dirichlet condition when $\kappa \geq 100$.

\subsection{Effect of vertical flow}

Dimensionless parameter $\kappa_{z} \quad$ (i.e., $\left.\quad K_{z} d^{2} /\left(K_{x} B^{2}\right)\right)$ dominates the effect of vertical flow on transient head distributions at an observation point. Consider $\kappa_{1} \bar{d}_{1}=\kappa_{2} \bar{d}_{2}=\kappa_{3} \bar{d}_{3}=\kappa_{4} \bar{d}_{4}=100$ for lateral aquifer boundaries under the Dirichlet condition as discussed in Sect. 3.1. The temporal distributions of $\bar{h}$ predicted by the present solution, Eq. (30a), with $\kappa_{z}=0.01,0.1,1$, and 10 are demonstrated in Fig. 3. The temporal distribution of $\bar{h}$ predicted by the Manglik and Rai (1998) solution based on 2-D flow without the vertical component is taken in order to address the effect of vertical flow. The figure reveals that $\bar{h}$ increases with $\kappa_{z}$ when $\kappa_{z} \leq 1$. The difference in $\bar{h}$ predicted by both solutions indicates the vertical flow effect. The Manglik and Rai (1998) solution obviously overestimates the head. The vertical flow prevails, and its effect should be taken into account when $\kappa_{z}<1$, indicating a thick aquifer, a small ratio of $K_{z} / K_{x}$, and/or an observation point near a recharge region. On the other hand, the present solution for the cases of $\kappa_{z}=1$ and 10 agrees well with Manglik and Rai (1998) solution, indicating that the vertical flow effect is ignorable when $\kappa_{z} \geq 1$. We can recognize from the agreement that existing solutions neglecting the vertical flow effect give good predictions when $\kappa_{z} \geq 1$.

\subsection{Effect of specific storage}

Some of the existing models use the Laplace equation as a governing equation by assuming $S_{\mathrm{s}}=0$ (e.g., Singh, 1976; Schmitz and Edenhofer, 1988; Zlotnik and Ledder, 1993). The assumption is valid when $\varepsilon$ (i.e., $S_{\mathrm{y}} /\left(S_{\mathrm{s}} B\right)$ ) is larger than a certain value. This section quantifies the value. The Zlotnik and Ledder (1993) model based on the 3-D Laplace equa- 

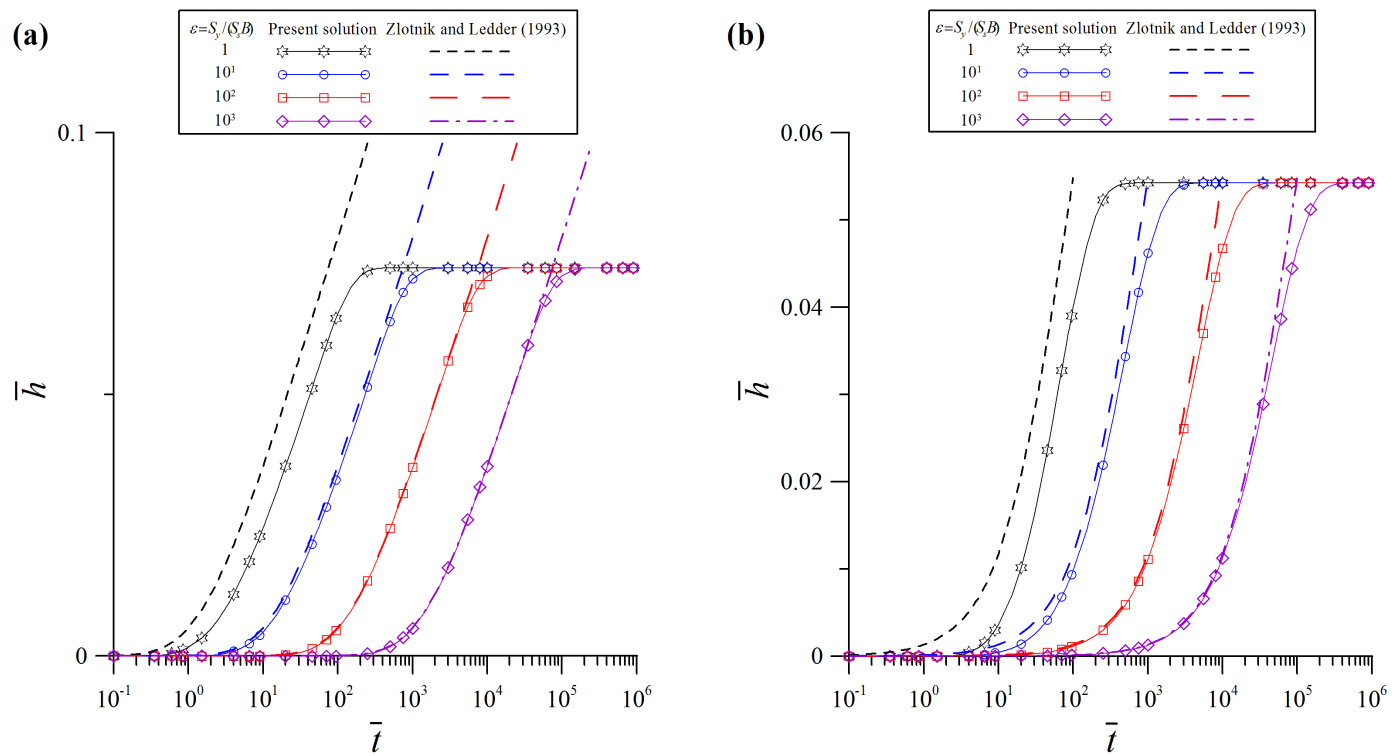

Figure 4. Temporal distributions of the dimensionless head for (a) $\kappa_{z}=10$ and (b) $\kappa_{z}=10^{-2}$ predicted by the Zlotnik and Ledder (1993) solution based on the assumption of $S_{\mathrm{S}}=0$ and the present solution relaxing the assumption.

tion, Eq. (1) with $S_{\mathrm{s}}=0$, is taken for comparison with the present model using Eq. (1) with $S_{\mathrm{s}} \neq 0$. The dimensionless variables of $s, x, y, z, t, X$, and $Y$ in their model are replaced by $\bar{h} / \xi,\left(\kappa_{z}\right)^{1 / 2} \bar{x},\left(\kappa_{z}\right)^{1 / 2} \bar{y}, \bar{z}, \kappa_{z} \bar{t} / \varepsilon,\left(\kappa_{z}\right)^{1 / 2} \bar{a}$, and $\left(\kappa_{z}\right)^{1 / 2} \bar{b}$, respectively, for ease of comparisons. Consider the cases of $\kappa_{z}=10^{-2}$ for an observation point located at a 3-D flow area and $\kappa_{z}=10$ for the point located at a 2-D flow area as discussed in Sect. 3.2. The assumption can be assessed through the comparison in the dimensionless heads predicted by both solutions for $\varepsilon=1,10,10^{2}$, and $10^{3}$ as shown in Fig. $4 \mathrm{a}$ for $\kappa_{z}=10$ and Fig. $4 \mathrm{~b}$ for $\kappa_{z}=10^{-2}$. The present solution predicts a steady-state $\bar{h}$ of 0.074 in Fig. 4 a and 0.054 in Fig. 4b after certain times due to lateral Dirichlet boundaries (i.e., $\kappa_{1} \bar{d}_{1}=\kappa_{2} \bar{d}_{2}=\kappa_{3} \bar{d}_{3}=\kappa_{4} \bar{d}_{4}=100$ ) as discussed in Sect. 3.1. In contrast, their solution predicts $\bar{h}$, which increases with $\bar{t}$ due to the absence of lateral boundaries. When $\varepsilon=1$ and 10 , both solutions give different values of $\bar{h}$ for both cases of $\kappa_{z}=10^{-2}$ and $\kappa_{z}=10$ before $\bar{t}=100$, indicating that the assumption of $S_{\mathrm{s}}=0$ causes inaccurate $\bar{h}$. When $\varepsilon=10^{2}$ and $10^{3}$, both solutions predict very close results of $\bar{h}$ for both cases before the time of approaching steady-state $\bar{h}$. These results lead to the conclusion that the assumption of $S_{\mathrm{s}}=0$ is valid when $\varepsilon \geq 100$ for 3-D and 2-D flow cases.

\subsection{Transient recharge rate}

Most articles (e.g., Rai et al., 1998; Chang and Yeh, 2007; Illas et al., 2008; Bansal and Teloglou, 2013) define a transient recharge rate as $I_{t}(t)=I_{1}+I_{0} \exp (-r t)$ (i.e., $\xi_{t}(\bar{t})=\xi_{1}+\xi_{0} \exp (-\gamma \bar{t})$ for a dimensionless rate) where $\xi_{t}=I_{t} / K_{z}, \xi_{1}=I_{1} / K_{z}, \xi_{0}=I_{0} / K_{z}, \gamma=r S_{\mathrm{s}} d^{2} / K_{x}$, and $r$ is a decay constant. The rate exponentially declines

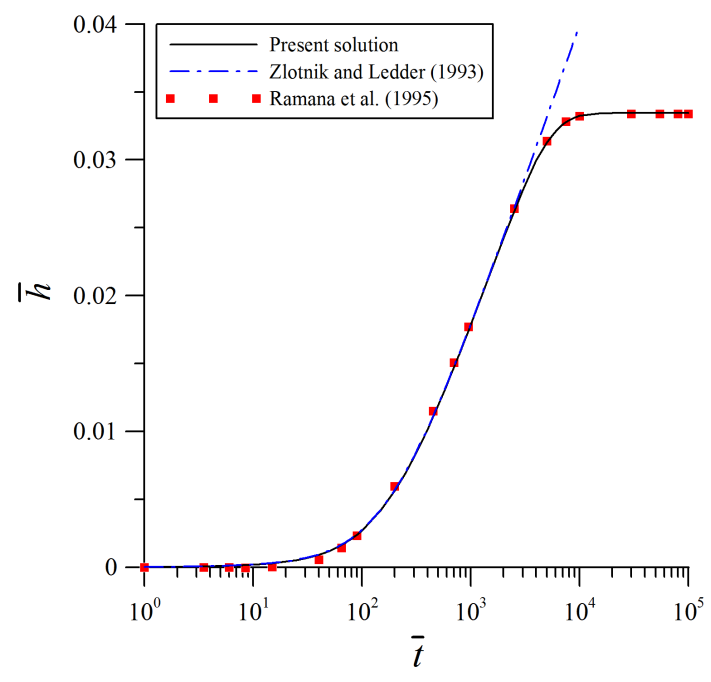

Figure 5. Temporal distributions of the dimensionless head subject to a transient recharge rate predicted by the Ramana et al. (1995) solution, the Zlotnik and Ledder (1993) solution, and the present solution with $\kappa_{z}=1, \kappa=100$, and $\varepsilon=100$.

from an initial value of $I_{1}+I_{0}$ to an ultimate value of $I_{1}$. In the present solution, Eq. (30a), can be applied for the response of the head to the transient rate based on Eq. (33). Substituting $\partial \xi_{t}(\tau) / \partial \tau=-\gamma \xi_{0} \exp (-\gamma \tau)$ into Eq. (33) and integrating the result for $\tau$ from $\tau=0$ to $\tau=\bar{t}$ yields $\bar{h}_{I 0}$ plus Eq. (30a), where $\xi$ in Eq. (30a), $\phi_{m, n}$ in Eq. (30b), $\exp \left(-\gamma_{0, m, n} \bar{t}\right)$ in Eq. (30c), and $\exp \left(-\gamma_{j, m, n} \bar{t}\right)$ in Eq. (30d) are replaced by $\xi_{0}, \phi_{m, n}[\exp (-\gamma \bar{t})-1]$, $\gamma\left[\exp (-\gamma \bar{t})-\exp \left(\gamma_{0, m, n} \bar{t}\right)\right] /\left(\gamma_{0, m, n}+\gamma\right)$, and $\gamma[\exp (-\gamma$ 

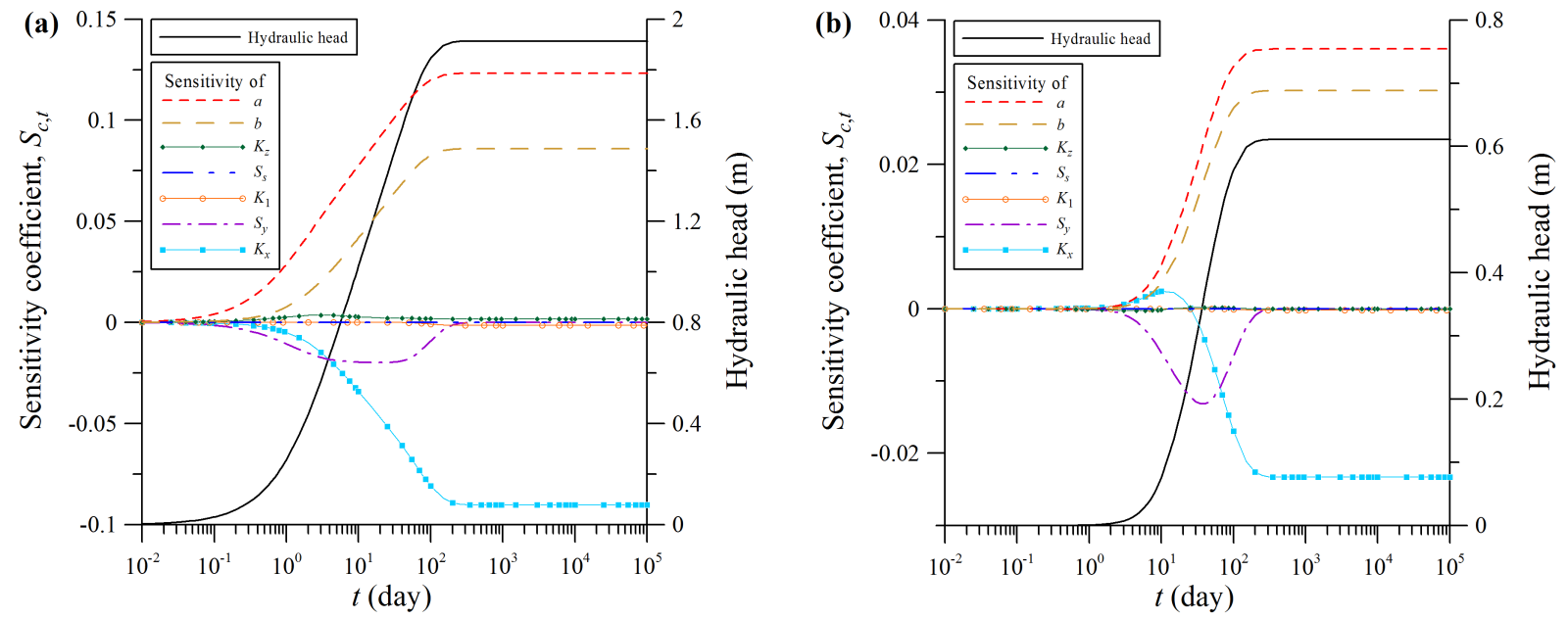

Figure 6. Temporal distributions of the normalized sensitivity coefficients of the hydraulic head at the observation points of (a) ( $x$, $y$, $z)=(555,500,-10)$ and $(\mathbf{b})(x, y, z)=(800,500,-10)$ to the changes in parameters $a, b, K_{z}, S_{\mathrm{s}}, K_{1}, S_{\mathrm{y}}$, and $K_{x}$.

$\left.\bar{t})-\exp \left(\gamma_{j, m, n} \bar{t}\right)\right] /\left(\gamma_{j, m, n}+\gamma\right)$, respectively. Similarly, the Zlotnik and Ledder (1993) solution can also be used to obtain the head subject to the transient rate by substituting it into Eq. (33) and then integrating the result using numerical approaches. Now, we consider the Ramana et al. (1995) solution depicting 2-D flow induced by the transient rate in rectangular aquifers with the lateral boundaries under the Dirichlet condition. Figure 5 shows the temporal distributions of $\bar{h}$ for the transient rate predicted by these three solutions when $\kappa_{z}=1, \kappa=100$, and $\varepsilon=100$. The present solution agrees well with the Ramana et al. (1995) solution. We can recognize from the agreement that, even for transient rates, the Robin condition reduces to the Dirichlet condition when $\kappa \geq 100$ (i.e., $\kappa_{1} \bar{d}_{1}=\kappa_{2} \bar{d}_{2}=\kappa_{3} \bar{d}_{3}=\kappa_{4} \bar{d}_{4}=100$ ) as discussed in Sect. 3.1 and the vertical flow effect is ignorable when $\kappa_{z} \geq 1$ as discussed in Sect. 3.2. Moreover, agreement on $\bar{h}$ estimated by the present solution and the Zlotnik and Ledder (1993) solution before $\bar{t}=3 \times 10^{3}$ will make it clear that, even for transient rates, assuming aquifer incompressibility (i.e., $S_{\mathrm{S}}=0$ ) is valid when $\varepsilon \geq 100$ as discussed in Sect. 3.3.

\subsection{Sensitivity analysis}

Consider point $\mathrm{A}$ of $(555,500,-10 \mathrm{~m})$ at a $3-\mathrm{D}$ flow region (i.e., $\left.\kappa_{z}<1\right)$ and point $\mathrm{B}$ of $(800,500,-10 \mathrm{~m})$ at a 2-D flow region (i.e., $\kappa_{z} \geq 1$ ) as discussed in Sect. 3.2. Localized recharge distributes over the square area of $450 \mathrm{~m} \leq x \leq 550 \mathrm{~m}$ and $450 \mathrm{~m} \leq y \leq 550 \mathrm{~m}$. The distance $d$ herein is set to $5 \mathrm{~m}$ for point $\mathrm{A}$ and $250 \mathrm{~m}$ for point $\mathrm{B}$. The aquifer system is of isotropy with $K_{x}=K_{y}$ and symmetry with $K_{1}=K_{2}=K_{3}=K_{4}$ for conciseness. To investigate the responses of the hydraulic heads at these two points to the change in each of $a, b, S_{\mathrm{s}}, S_{\mathrm{y}}, K_{x}$ (or $K_{y}$ ), $K_{z}$, and $K_{1}$ (or $K_{2}, K_{3}$, and $K_{4}$ ), the sensitivity analysis is performed by Eq. (36). The curves of the normalized sensitivity coefficient $S_{c, t}$ versus $t$ for these seven parameters are shown in Fig. $6 \mathrm{a}$ for point $\mathrm{A}$ and Fig. $6 \mathrm{~b}$ for point B. The figure shows that the hydraulic heads at both points are more sensitive to the changes in $a, b, K_{x}$, and $S_{\mathrm{y}}$ than those in the others. This may indicate that a flow model should include at least these four parameters. The figure also shows that the heads at points $\mathrm{A}$ and B are insensitive to the change in $K_{1}$ because of $\kappa_{1} \bar{d}_{1}=4500>100$ as discussed in Sect. 3.1. In addition, $S_{c, t}$ to $K_{z}$ for point A is nonzero after $t=0.4$ day due to $\kappa_{z}=6.25 \times 10^{-3}<1$ as discussed in Sect. 3.2. In contrast, $S_{c, t}$ to $K_{z}$ for point B is very close to zero over the entire period because of $\kappa_{z}=15.625>1$. Moreover, the heads at points $\mathrm{A}$ and B are insensitive to the change in $S_{\mathrm{S}}$ due to $\varepsilon=500>100$ as discussed in Sect. 3.3.

\section{Conclusions}

A mathematical model is developed to depict spatiotemporal head distributions induced by localized recharge with an arbitrary time-varying rate in a rectangular unconfined aquifer bounded by Robin boundaries with different hydraulic parameters. A governing equation for 3-D flow is considered. A first-order free-surface equation with a source term representing the recharge is employed for describing the water table movement. The analytical head solution of the model is obtained by applying the Laplace transform, the doubleintegral transform, and Duhamel's theorem. The use of rectangular aquifer domain leads to two merits. One is that the integration for the solution to the depth-average head can be analytically done. The other is that existing solutions based on aquifers of infinite extent are special cases of the present solution when the recharge time is less than the boundaryeffect time. The present solution is applicable under the con- 
ditions of aquifer homogeneity, $|h| / B<0.5, I / K_{z}<0.2$, and $\sigma B \geq 10^{3}$ due to Eq. (8a) neglecting the effect of unsaturated flow above water table (Marino, 1967; Tartakovsky and Neuman, 2007). The sensitivity analysis is performed to explore the response of the head to the change in each of hydraulic parameters. With the aid of the present solution, the following conclusions can be drawn:

1. With respect to affecting $\bar{h}$ at observation points, the Robin condition specified at $\bar{x}=0$ reduces to the Dirichlet condition when $\kappa_{1} \bar{d}_{1} \geq 100$ (i.e., $\left.K_{1} d_{1} /\left(K_{x} b_{1}\right) \geq 100\right)$ and no-flow condition when $\kappa_{1} \bar{d}_{1} \leq 10^{-2}$. The quantitative criteria for $\kappa_{1} \bar{d}_{1}$ are applicable to $\kappa_{2} \bar{d}_{2}, \kappa_{3} \bar{d}_{3}$, and $\kappa_{4} \bar{d}_{4}$ for the Robin conditions specified at $\bar{x}=\bar{l}, \bar{y}=0$, and $\bar{y}=\bar{w}$, respectively.
2. The vertical flow causes a significant decrease in the hydraulic head at an observation point when $\kappa_{z}<1$ (i.e., $\left.K_{z} d^{2} /\left(K_{x} B^{2}\right)<1\right)$. When $\kappa_{z} \geq 1$, the effect of vertical flow on the head is ignorable, and conventional models considering 2-D flow without the vertical component can therefore predict accurate results.

3. The 3-D Laplace equation based on the assumption of $S_{\mathrm{s}}=0$ can be regarded as a flow governing equation when $\varepsilon \geq 100$ (i.e., $\left.S_{\mathrm{y}} /\left(S_{\mathrm{s}} B\right) \geq 100\right)$ for the whole aquifer domain. Otherwise, head predictions based on the Laplace equation are overestimated.

4. The abovementioned conclusions are also applicable to problems of groundwater flow subject to recharge with arbitrary time-varying rates. 


\section{Appendix A: Derivation of Eq. (30a)}

We begin with function $G(p)$ from Eq. (28):

$G(p)=\frac{\cosh [(1+\bar{z}) \lambda]}{p\left(p \varepsilon \kappa_{z} \cosh \lambda+\kappa_{z} \lambda \sinh \lambda\right)}$,

with

$\lambda=\sqrt{\left(p+f_{m, n}\right) / \kappa_{z}}$,

where $f_{m, n}=\alpha_{m}^{2}+\kappa_{y} \beta_{n}^{2}$. Equation (A1) is a single-value function to $p$ in the complex plane because it satisfies $G\left(p^{+}\right)=G\left(p^{-}\right)$, where $p^{+}$and $p^{-}$are the polar coordinates defined, respectively, as

$p^{+}=r_{a} \exp (i \theta)-f_{m, n}$

and

$p^{-}=r_{a} \exp [i(\theta-2 \pi)]-f_{m, n}$,

where $r_{a}$ represents a radial distance from the origin at $p=-f_{m, n}, i=\sqrt{-1}$ is the imaginary unit, and $\theta$ is an argument between 0 and $2 \pi$. Substitute $p=p^{+}$in Eq. (A3) into Eq. (A2), and we have

$\lambda=\sqrt{r_{a} / \kappa_{z}} \exp (i \theta / 2)=\sqrt{r_{a} / \kappa_{z}}[\cos (\theta / 2)+i \sin (\theta / 2)]$.

Similarly, we can have

$$
\begin{aligned}
\lambda & =\sqrt{r_{a} / \kappa_{z}} \exp [i(\theta-2 \pi) / 2]=-\sqrt{r_{a} / \kappa_{z}}[\cos (\theta / 2) \\
& +i \sin (\theta / 2)]
\end{aligned}
$$

after $p$ in Eq. (A2) is replaced by $p^{-}$in Eq. (A4). Substitution of Eqs. (A3) and (A5) into Eq. (A1) yields the same result as that obtained by substituting Eqs. (A4) and (A6) into Eq. (A1), indicating that Eq. (A1) is a single-value function without branch cut and its inverse Laplace transform equals the sum of residues for poles in the complex plane.

The residue for a simple pole can be formulated as

$\operatorname{Res}=\lim _{p-\varphi} G(p) \exp (p \bar{t})(p-\varphi)$,

where $\varphi$ is the location of the pole of $G(p)$ in Eq. (A1). The function $G(p)$ has infinite simple poles at the negative part of the real axis in the complex plane. The locations of these poles are the roots of the following equation:

$p\left(p \varepsilon \kappa_{z} \cosh \lambda+\kappa_{z} \lambda \sinh \lambda\right)=0$,

which is obtained by setting the denominator in Eq. (A1) to zero. Obviously, one pole is at $p=0$, and its residue based on Eqs. (A1) and (A7) with $\lambda_{m, n}=\sqrt{f_{m, n} / \kappa_{z}}$ can be expressed as

$\phi_{m, n}=\cosh \left[(1+\bar{z}) \lambda_{m, n}\right] /\left(\kappa_{z} \lambda_{m, n} \sinh \lambda_{m, n}\right)$.
The locations of other poles of $G(p)$ are the roots of the following equation:

$p \varepsilon \kappa_{z} \cosh \lambda+\kappa_{z} \lambda \sinh \lambda=0$,

which is the expression in the parentheses in Eq. (A8). One pole is between $p=0$ and $p=-f_{m, n}$. Let $\lambda=\lambda_{0, m, n}$, and Eq. (A2) becomes $p=-f_{m, n}+\kappa_{z} \lambda_{0, m, n}^{2}$. Substituting $\lambda=\lambda_{0, m, n}, \quad p=-f_{m, n}+\kappa_{z} \lambda_{0, m, n}^{2}, \quad \cosh \lambda_{0, m, n}$ $=\left[\exp \lambda_{0, m, n}+\exp \left(-\lambda_{0, m, n}\right)\right] / 2, \quad$ and $\sinh \lambda_{0, m, n}=[\exp$ $\left.\lambda_{0, m, n}-\exp \left(-\lambda_{0, m, n}\right)\right] / 2$ into Eq. (A10) and rearranging the result leads to Eq. (32). The pole is at $p=-f_{m, n}+\kappa_{z} \lambda_{0, m, n}^{2}$ with a numerical value of $\lambda_{0, m, n}$. With Eq. (A1), Eq. (A7) equals

$\operatorname{Res}=\frac{\cosh [(1+\bar{z}) \lambda]}{p\left(p \varepsilon \kappa_{z} \cosh \lambda+\kappa_{z} \lambda \sinh \lambda\right)} \exp (p \bar{t})(p-\varphi)$.

Apply L'Hospital's rule to Eq. (A11), and then we have

Res $=\frac{-2 \lambda \cosh [(1+\bar{z}) \lambda]}{p\left[\left(1+2 \varepsilon \kappa_{z}\right) \lambda \cosh \lambda+(1-\varepsilon p) \sinh \lambda\right]} \exp (p \bar{t})$.

The residue for the pole at $p=-f_{m, n}+\kappa_{z} \lambda_{0, m, n}^{2}$ can be defined as

$\phi_{0, m, n}=\frac{-2 \lambda_{0, m, n} \cosh \left[(1+\bar{z}) \lambda_{0, m, n}\right] \exp \left(-\gamma_{0, m, n} \bar{t}\right)}{\gamma_{0, m, n}\left[\left(1+2 \varepsilon \kappa_{z}\right) \lambda_{0, m, n} \cosh \lambda_{0, m, n}+\left(1-\varepsilon \gamma_{0, m, n}\right) \sinh \lambda_{0, m, n}\right]}$,

which is obtained by Eq. (A12) with $\lambda=\lambda_{0, m, n}$ and $p=-f_{m, n}+\kappa_{z} \lambda_{0, m, n}^{2}=-\gamma_{0, m, n}$. On the other hand, infinite poles behind $p=-f_{m, n}$ are at $p=\gamma_{j, m, n}$, where $j \in 1,2,3, \ldots \infty$. Let $\lambda=\sqrt{-1} \lambda_{j, m, n}$, and Eq. (A2) yields $p=-f_{m, n}-\kappa_{z} \lambda_{j, m, n}^{2}$. Substituting $\lambda=\sqrt{-1} \lambda_{j, m, n}$, $p=-f_{m, n}-\kappa_{z} \lambda_{j, m, n}^{2}, \cosh \left(\sqrt{-1} \lambda_{j, m, n}\right)=\cos \lambda_{j, m, n}$, and $\sinh \left(\sqrt{-1} \lambda_{j, m, n}\right)=\sqrt{-1} \sin \lambda_{j, m, n}$ into Eq. (A10) and rearranging the result gives rise to Eq. (31). These poles are at $p=-f_{m, n}-\kappa_{z} \lambda_{j, m, n}^{2}$ with numerical values of $\lambda_{j, m, n}$. On the basis of Eq. (A12) with $\lambda=\sqrt{-1} \lambda_{j, m, n}$ and $p=-f_{m, n}-\kappa_{z} \lambda_{j, m, n}^{2}=-\gamma_{j, m, n}$, the residues for these poles at $p=-f_{m, n}-\kappa_{z} \lambda_{j, m, n}^{2}$ can be expressed as

$\phi_{j, m, n}=\frac{-2 \lambda_{j, m, n} \cos \left[(1+\bar{z}) \lambda_{j, m, n}\right] \exp \left(-\gamma_{j, m, n} \bar{t}\right)}{\gamma_{j, m, n}\left[\left(1+2 \varepsilon \kappa_{z}\right) \lambda_{j, m, n} \cos \lambda_{j, m, n}+\left(1-\varepsilon \gamma_{j, m, n}\right) \sin \lambda_{j, m, n}\right]}$

As a result, the inverse Laplace transform for Eq. (A1) is the sum of Eqs. (A9) and (A13) and a simple series expended in the RHS function in Eq. (A14) (i.e., $\phi_{m, n}+\phi_{0, m, n}+\sum_{j=1}^{\infty} \phi_{j, m, n}$ ). Finally, Eq. (30a) can be derived after taking the inverse double-integral transform for the result using the following formula (Latinopoulos, 1985, Eq. 14):

$$
\begin{gathered}
\bar{h}(\bar{x}, \bar{y}, \bar{z}, \bar{t})=\xi \sum_{m=1}^{\infty} \sum_{n=1}^{\infty}\left(\phi_{m, n}+\phi_{0, m, n}+\sum_{j=1}^{\infty} \phi_{j, m, n}\right) \\
F_{m} F_{n} U_{m} U_{n},
\end{gathered}
$$

where $\xi$ and $U_{m} U_{n}$ result from $\xi U_{m} U_{n}$ in Eq. (28). 


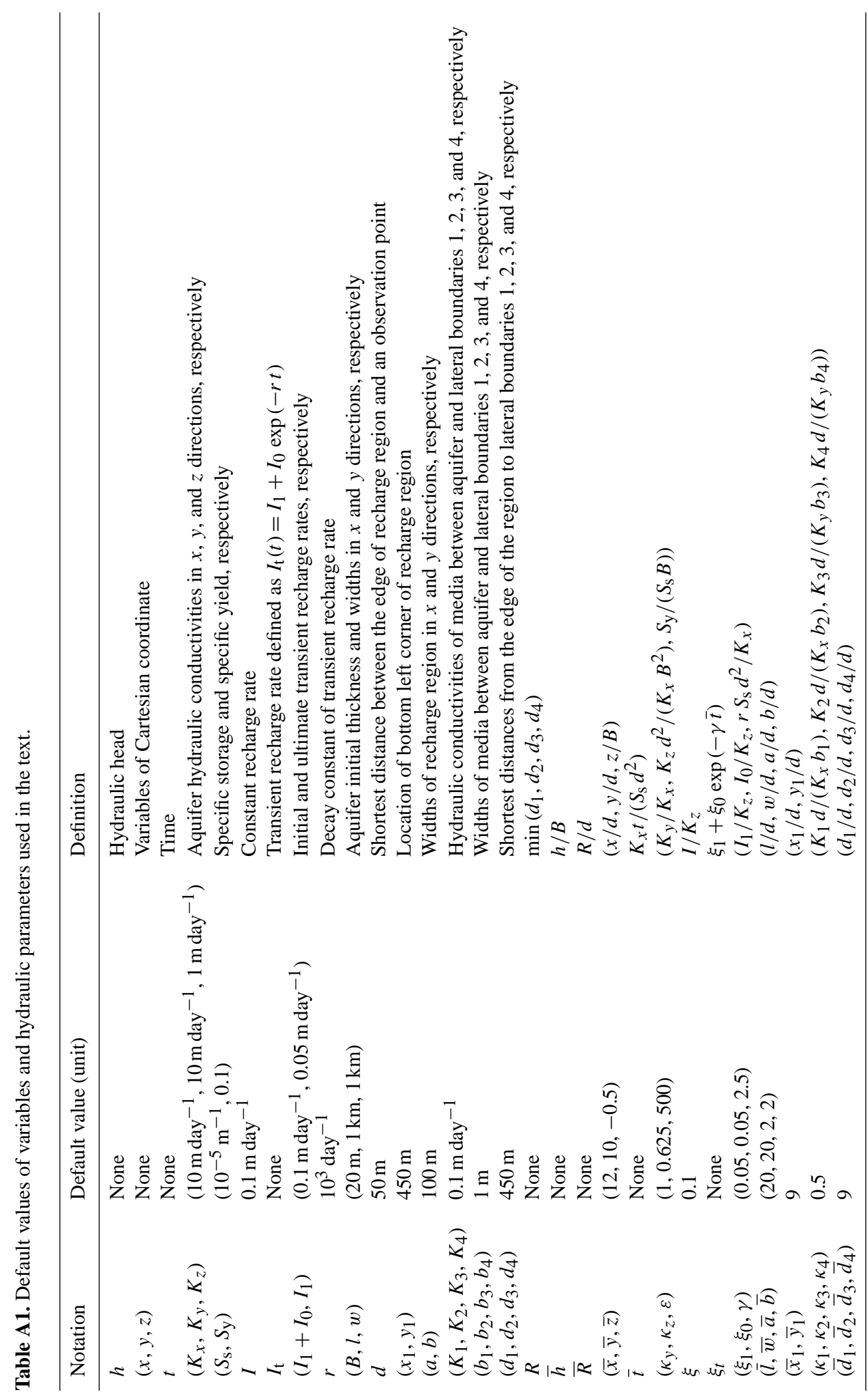


Acknowledgements. This study has been partly supported by the Taiwan Ministry of Science and Technology under the grants MOST 103-2221-E-009-156 and MOST 104-2221-E-009148-MY2. The computer software used to generate the results in Figs. 2-6 is available upon request. The authors would like to thank the editor, Alberto Guadagnini, and two reviewers for their valuable and constructive comments.

Edited by: A. Guadagnini

\section{References}

Bansal, R. K. and Das, S. K.: Analytical Study of Water Table Fluctuation in Unconfined Aquifers due to Varying Bed Slopes and Spatial Location of the Recharge Basin, J. Hydrol. Eng., 15, 909917, 2010.

Bansal, R. K. and Teloglou, I. S.: An Analytical Study of Groundwater Fluctuations in Unconfined Leaky Aquifers Induced by Multiple Localized Recharge and Withdrawal, Global Nest J., 15, 394-407, 2013.

Bear, J.: Hydraulics of Groundwater, McGraw-Hill, New York, p. $158,1979$.

Bruggeman, G. A.: Analytical Solutions of Geohydrological Problems, Elsevier, the Netherlands, p. 326, 1999.

Chang, Y. C. and Yeh, H. D.: Analytical solution for groundwater flow in an anisotropic sloping aquifer with arbitrarily located multiwells, J. Hydrol., 347, 143-152, 2007.

Chor, T. L. and Dias, N. L.: Technical Note: A simple generalization of the Brutsaert and Nieber analysis, Hydrol. Earth Syst. Sci., 19, 2755-2761, doi:10.5194/hess-19-2755-2015, 2015.

Dagan, G.: Linearized solutions of free surface groundwater flow with uniform recharge, J. Geophys. Res., 72, 1183-1193, 1967.

Hansen, B. P. and Lapham, W. W.: Geohydrology and simulated ground-water flow, Plymouth-Carver Aquifer, southeastern Massachusetts, Report 90-4204, US Geological Survey, USA, 1-69, 1992.

Hantush, M. S.: Growth of a ground water ridge in response to deep percolation, Symposium on Transient Ground water Hydraulics, 25-27 July 1963, Ft. Collins Colorado, 1963.

Hantush, M. S.: Growth and decay of groundwater-mounds in response to uniform percolation, Water Resour. Res., 3, 227-234, doi:10.1029/WR003i001p00227, 1967.

Hsieh, P. C., Hsu, H. T., Liao, C. B., and Chiueh, P. T.: Groundwater response to tidal fluctuation and rainfall in a coastal aquifer, J. Hydrol., 521, 132-140, 2015.

Illas, T. S., Thomas, Z. S., and Andreas, P. C.: Water table fluctuation in aquifers overlying a semi-impervious layer due to transient recharge from a circular basin, J. Hydrol., 348, 215-223, 2008.

Ireson, A. M. and Butler, A. P.: A critical assessment of simple recharge models: application to the UK Chalk, Hydrol. Earth Syst. Sci., 17, 2083-2096, doi:10.5194/hess-17-20832013, 2013.

Latinopoulos, P.: Analytical Solutions for Periodic Well Recharge in Rectangular Aquifers with 3rd-Kind Boundary-Conditions, J. Hydrol., 77, 293-306, 1985.
Latinopoulos, P.: Analytical Solutions for Strip Basin Recharge to Aquifers with Cauchy Boundary-Conditions, J. Hydrol., 83, 197-206, 1986.

Liang, X. Y. and Zhang, Y.-K.: Analyses of uncertainties and scaling of groundwater level fluctuations, Hydrol. Earth Syst. Sci., 19, 2971-2979, doi:10.5194/hess-19-2971-2015, 2015.

Liang, X. Y., Zhang, Y. K., and Schilling, K. E.: Analytical solutions for two-dimensional groundwater flow with subsurface drainage tiles, J. Hydrol., 521, 556-564, 2015.

Manglik, A. and Rai, S. N.: Two-Dimensional Modelling of Water Table Fluctuations due to Time-Varying Recharge from Rectangular Basin, Water Resour. Manage., 12, 467-475, 1998.

Manglik, A., Rai, S. N., and Singh, R. N.: Response of an Unconfined Aquifer Induced by Time Varying Recharge from a Rectangular Basin, Water Resour. Manage., 11, 185-196, 1997.

Marino, M. A.: Hele-Shaw model study of the growth and decay of groundwater ridges, J. Geophys. Res., 72, 1195-1205, doi:10.1029/JZ072i004p01195, 1967.

Neuman, S. P.: Effect of partial penetration on flow in unconfined aquifers considering delayed gravity response, Water Resour Res., 10, 303-312, doi:10.1029/WR010i002p00303, 1974.

Ostendorf, D. W., DeGroot, D. J., and Hinlein, E. S.: Unconfined aquifer response to infiltration basins and shallow pump tests, J. Hydrol., 338, 132-144, 2007.

Rai, S. N., Ramana, D. V., and Singh, R. N.: On the Prediction of Ground-Water Mound Formation in Response to Transient Recharge from a Circular Basin, Water Resour. Manage., 12, 271-284, 1998.

Rai, S. N., Manglik, A., and Singh, V. S.: Water table fluctuation owing to time-varying recharge, pumping and leakage, J. Hydrol., 324, 350-358, 2006.

Ramana, D. V., Rai, S. N., and Singh, R. N.: Water table fluctuation due to transient recharge in a 2-D aquifer system with inclined base, Water Resour. Manage., 9, 127-138, doi:10.1007/BF00872464, 1995.

Rao, N. H. and Sarma, P. B. S.: Growth of Groundwater Mound in Response to Recharge, Ground Water, 18, 587-595, 1980.

Rao, N. H. and Sarma, P. B. S.: Recharge to Aquifers with Mixed Boundaries, J. Hydrol., 74, 43-51, 1984.

Schmitz, G. and Edenhofer, J.: Semi Analytical Solution for the Groundwater Mound Problem, Adv Water. Resour., 11, 21-24, 1988.

Singh, R.: Prediction of mound geometry under recharge basins, Water Resour. Res., 12, 775-780, doi:10.1029/WR012i004p00775, 1976.

Singh, S. K.: Rate and volume of stream flow depletion due to unsteady pumping, J. Irrig. Drain. E-Asce, 131, 539-545, 2005.

Tartakovsky, G. D. and Neuman, S. P.: Three-dimensional saturated-unsaturated flow with axial symmetry to a partially penetrating well in a compressible unconfined aquifer, Water. Resour. Res., 43, W01410, doi:10.1029/2006WR005153, 2007.

van der Spek, J. E., Bogaard, T. A., and Bakker, M.: Characterization of groundwater dynamics in landslides in varved clays, Hydrol. Earth Syst. Sci., 17, 2171-2183, doi:10.5194/hess-172171-2013, 2013.

Wang, C. T. and Yeh, H. D.: Obtaining the steady-state drawdown solutions of constant-head and constant-flux tests, Hydrol. Process., 22, 3456-3461, 2008. 
Yeh, H. D. and Chang, Y. C.: Recent advances in modeling of well hydraulics, Adv. Water Resour., 51, 27-51, 2013.

Yeh, H. D., Chang, Y. C., and Zlotnik, V. A.: Stream depletion rate and volume from groundwater pumping in wedge-shape aquifers, J. Hydrol., 349, 501-511, 2008.
Zlotnik, V. and Ledder, G.: Groundwater-Flow in a Compressible Unconfined Aquifer with Uniform Circular Recharge, Water Resour. Res., 28, 1619-1630, 1992.

Zlotnik, V. and Ledder, G.: Groundwater Velocity in an Unconfined Aquifer with Rectangular Areal Recharge, Water Resour. Res., 29, 2827-2834, 1993. 